\title{
Annual colonoscopy, chest radiography, and computed tomography of the liver did not prolong survival in patients with colorectal cancer
}

Schoemaker D, Black $R$, Giles L, et al. Yearly colonoscopy, liver CT, and chest radiography do not influence 5-year survival of colorectal cancer patients. Gastroenterology 1998 fan;114:7-14.

\section{Question}

Compared with standard follow up, can intensive follow up (ie, the addition of annual colonoscopy, chest radiography, and computed tomography of the liver) increase the 5 year survival rate in patients who have been treated for colorectal cancer?

\section{Design}

Randomised controlled trial with 5 years of follow up.

\section{Setting}

Australia.

\section{Patients}

325 patients (age range 29 to $84 \mathrm{y}, 64 \%$ men) who were scheduled to receive curative resections for newly diagnosed colorectal cancer. Of 238 patients with colon cancer, $17 \%$ had Dukes' A stage, $51 \%$ had Dukes' B stage, and 32\% had Dukes' C stage; of 87 patients with rectal cancer, 35.5\% had Dukes' A stage, 35.5\% had Dukes' B stage, and $29.0 \%$ had Dukes' C stage. Patients were excluded if they were unlikely to complete 5 years of follow up, lived in a remote area, were $>85$ years of age, refused to participate in the trial, or had evidence of residual or distant malignancy. Follow up was $94 \%$.

\section{Intervention}

After stratification for the site and stage of the tumour, patients were allocated to receive intensive follow up $(n=167)$ or standard follow up $(\mathrm{n}=158)$. Standard follow up comprised a regular clinical review (history, examination, and screening investigations) every 3 months for 2 years and every 6 months thereafter; intensive follow up comprised standard follow up plus annual chest radiography, computed tomography of the liver, and colonoscopy.

\section{Main outcome measure}

5 year survival rate.

\section{Main results}

No difference in the survival rate existed at 5 years between patients who received intensive follow up and those who received standard follow up (table). The study had $90 \%$ power to detect a $15 \%$ difference at the 0.05 level of significance. Cox regression models showed that Dukes' staging and tumour adherence to other structures influenced survival in patients with colorectal cancer. No difference in survival between follow up schedules existed after controlling for these variables.

\section{Conclusion}

The addition of annual colonoscopy, computed tomography of the liver, and chest radiography to symptom and simple screening follow up did not increase the 5 year survival rate for patients with colorectal cancer.

Sources of funding: Anti-Cancer Foundation of the Universities of South Australia and the Commonwealth Health and Medical Research Council of the Department of Veteran's Affairs.

For article reprint: Dr 7 Toouli, Gastrointestinal Surgical Unit, Flinders Medical Cen-

Table 1 Intensive follow up $v$ standard follow up for patients with colorectal cancer

\begin{tabular}{llllll}
\hline Outcome at 5 years & $\begin{array}{c}\text { Intensive } \\
\text { follow up }\end{array}$ & $\begin{array}{c}\text { Standard } \\
\text { follow up }\end{array}$ & RBI* & $95 \%$ CI & NNT† \\
\hline Survivors & $73 \%$ & $63 \%$ & $15 \%$ & -2 to 35 & NS $\ddagger$
\end{tabular}

${ }^{\star} \mathrm{RBI}=$ relative benefit increase (the proportional increase in the rates of good events between the intensive and standard follow up groups); calculated from data in the article.

$\dagger \mathrm{NNT}=$ number needed to treat (the number of patients who need to receive intensive follow up to achieve 1 additional favourable outcome); calculated from data in article.

$\ddagger \mathrm{NS}=$ not significant.

\section{Commentary}

Does the addition of annual computed tomography (CT) scanning, chest $x$ ray and colonoscopy to standard clinical follow up improve outcome in unselected patients with colorectal cancer? Sadly, the answer seems to be "no". This study and that of Makela and coworkers ${ }^{1}$ show no benefit in intensive follow up, and that of Ohlsson and colleagues ${ }^{2}$ showed no benefit in follow up at all.

Tumour stage and adherence to adjacent structures influenced survival, but when these were corrected for, and despite a $90 \%$ power to detect a $15 \%$ difference at $\mathrm{p}<0.05$, the 5 -year survival rate was not affected by intensive follow up. There was a $9 \%$ absolute increment in 5-year survival in the intensive group, but at least 600 patients in each group would be needed to show whether this is real. Briefly, the benefit of the 633 chest $x$ rays in those screened yearly was a single patient who survived 5 years. Annual colonoscopy showed only one asymptomatic curable metachronous lesion, at a cost of 505 extra colonoscopies in the intensive group. 608 more liver CT scans in the intensive group detected only one asymptomatic patient with metastases who may have benefited from liver surgery.

Carcinoembryonic antigen (CEA) is commonly used to look for early evidence of recurrence, though there is little evidence to support the practice. Raised CEA in Schoemaker $e$ al's group of patients was previously shown to be the first indicator of liver metastases in $80 \%$, and precededother test abnormalities by a median of 6 months, ${ }^{3}$ but raised CEA concentrations were - probably rightly - not taken as an indication for investigation in the current study. Although not a randomised trial, the recent data of Wolf and Cohen ${ }^{4}$ at the Sloan-Kettering Cancer Centre suggests that the survival advantage of early investigation and surgery triggered by rising CEA concentrations is probably less than $5 \%$.

Whatever else may now be done, follow up by routine imaging does not seem to improve outcome for unselected patients with colorectal cancer after surgery for cure, never mind the cost. We need to know for sure whether outcome is improved by CEA triggered treatments of any kind, or by any follow up of asymptomatic patients after apparently curative surgery for colorectal cancer.

P FAIRCLOUGH Department of Gastroenterology, Royal Hospitals NHS Trust, London, UK B HAYNES Health Information Research Unit, Department of Clinical Epidemiology and Biostatistics, McMaster University, Hamilton, Ontario, Canada

1 Makela JT, Laitenen SO, Kairoluoma MI. Five-year follow-up after radical surgery for colorectal cancer. Arch Surg 1996;130: 1062-7.

2 Ohlsson B, Breland U, Ekberg $\mathrm{H}$, et al. Follow-up after curative surgery for colorectal carcinoma. Randomised comparison with no follow-up. Dis Colon Rectum 1995;38:219-26.

3 McCall JL, Black RB, Rich CA, et al. The value of serum carcinoembryonic antigen in predicting recurrent disease following curative resection of colorectal cancer. Dis Colon Rectum tion of colorectal cancer. Dis Colon Rectum 1994;37:875-81

4 Wolf RF, Cohen AM. The miniscule benefit of serial carcinoembryonic antigen monitoring after effective curative resection for primary colorectal cancer. F Am Coll Surg 1997;185:60-4. 